\title{
How to structure an unstructured activity: generating physics rules from simulation or contrasting cases
}

\author{
Shima Salehi ${ }^{1}$, Martin Keil ${ }^{1}$, Eric Kuo ${ }^{1,2}$ and Carl Wieman ${ }^{1,2}$ \\ ${ }^{1}$ Stanford University, Graduate School of Education, 485 Lasuen Mall, Stanford, CA, 94305 US \\ ${ }^{2}$ Stanford University, Physics Department, 382 Via Pueblo, Stanford, CA, 94305 US
}

\begin{abstract}
Studies show that having students attempt to invent a scientific rule ("invention activity") before receiving direct instruction benefits their learning. However, the design affordances of these invention activities have not been fully addressed. In this paper, we compare the effect of two different brief invention activities on student learning about buoyancy. In one treatment condition, students are provided with contrasting cases of a phenomenon to invent the rule. In the other treatment condition, students explore the phenomenon using an interactive simulation. Students in the contrasting cases condition invented more complete rules and performed significantly better in solving buoyancy problems. We hypothesize that this difference between conditions results from different levels of attention to important features of the phenomenon, which we illustrate with differences in what students explore in the simulation. This study suggests that proper scaffolding to ensure sufficient exposure to the underlying structure is essential in designing an invention activity.
\end{abstract}

PACS: $01.40 . \mathrm{Fk}, 01.50 . \mathrm{ht}$

\section{INTRODUCTION}

Understanding how to design an invention activity is important. This study evaluates the effect of inventing from two types of materials on student understanding of a physical rule.

In a well-tested approach, students invent rules from a set of pre-designed, contrasting cases. By generating a rule to explain a range of cases, students can come to see the important features any sufficient rule needs to capture. These students are subsequently better prepared for future learning from direct instruction of these rules [1]. For example, results of previous studies have shown that inventing rules for characterizing variance or density in a set of examples improved students' ability to transfer those ideas to new contexts after subsequent instruction $[1,2]$.

An alternative approach is to have students invent explanations through exploration with an interactive computer simulation. The computer simulation gives students an unconstrained environment to generate whatever cases they want [3].

These approaches trade off on two important features. The contrasting cases are designed to show the full range of important features to a correct rule, but do not give students a chance to explore the phenomenon more generally or to create cases to test hypotheses. The simulation is designed to give students a tool to explore and make sense of a phenomenon on their own terms, but does not ensure that students will explore all the important aspects of the phenomenon on their own. The key question, which informs design of such invention activities, is how the trade-off between seeing the full range of variation and having agency to generate cases and test hypotheses affects student learning of physics rules.

\section{METHODS}

To explore this question, we recruited 79 community college students who received credit in their social science course for their participation. For the physics content of the study, we chose buoyancy — the mechanism underlying the sinking and floating of objects in fluids - as it has a balance of familiarity and non-intuitive complexity. Participants were prompted to invent a general rule that could predict whether an object placed in a fluid would sink or float (the correct "density rule" being that an object will float if the object's density is less than the fluid density). We randomly assigned participants to one of the following conditions: (a) Treatment $1(\mathrm{CC}, n=25)$ : contrasting cases as an invention activity + direct instruction, (b) Treatment 2 (SIM, $n=28$ ): interactive simulation as an invention activity + direct instruction, or (c) Control (CTRL, $n=26)$ : only direct instruction.

\section{A. Procedure \& Materials}

Participants worked for about an hour individually, with little guidance from the researchers. Figure 1 illustrates the sequence of activities for the three different conditions.

First, each participant completed a pre-test in 5 minutes. Participants in the treatment conditions (CC and SIM) received the corresponding training for their invention activity for 5 minutes. Then, they had 15 minutes to complete the invention activity. After the activity, in the next 5 minutes, they took a mid-test and engagement survey. After finishing the survey, they read an instructional 
text for 15 minutes, and then they had 15 minutes to answer the post-test. Participants in the control condition were directed to read the instructional text immediately after the pre-test. The timing of instructional text and post-test was the same for the control and the treatment conditions. By comparing CC or SIM to CTRL, we can evaluate the added benefits of either activity for subsequent learning from the text.

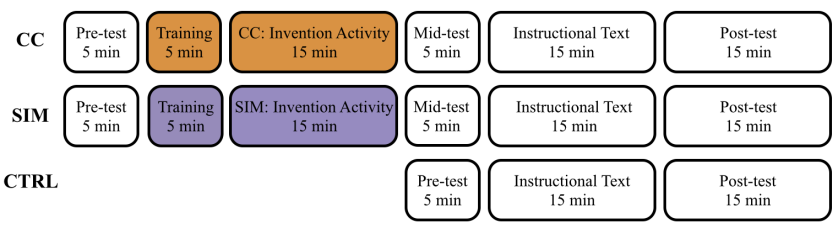

FIG 1. The design of the study across the three conditions.

\section{Training for invention activity}

Before starting the invention activity, participants in the treatment conditions received training in doing an invention activity (materials adopted from Hallinen [4]). In the CC condition, they were provided with six different cases of a balance scale that had a different mass at a different distance from the pivot on each side. Participants attempted to invent a general rule that would predict whether the scale would tip left, tip right, or be balanced. Then, they were told the correct rule ("the balance beam will tip to the side on which the mass $\mathrm{x}$ distance is greater"). In the SIM condition, participants received almost the same training; the only difference was that they observed a screen capture video of generating the six cases using an interactive simulation.

\section{Contrasting cases invention activity}

Participants were provided with six cases that represented different scenarios for sinking and floating of objects in fluids. Each case showed a cube in a fluid. Cases varied across three dimensions: 1 . the cube characteristics (i.e. mass and/or volume), 2. the fluid type, and 3. the buoyant behavior of the cube (i.e. sinking, floating, or suspended in the fluid). These cases were generated by taking screenshots of the PhET buoyancy simulation [5], the same simulation used in the SIM condition. The cases explicitly listed the values for the mass of the cube, volume of the cube, density of the cube, and density of the fluid. The task for participants was to invent a rule for floating and sinking of objects in fluids.

\section{Interactive simulation invention activity}

For the SIM condition, participants were directed to invent the same rule with the Buoyancy PhET simulation. In the simulation, participants could place a cube in a fluid and observe whether it would sink or float. The mass of the cube, volume of the cube, and type of fluid could be directly changed. The simulation also gave the same numerical values as in $\mathrm{CC}$.

\section{Instructional text}

As the direct instruction, participants received a fivepage instructional text describing the principles of buoyancy. The primary learning goal for the passage was to understand the density rule for floating and sinking, the rule participants were prompted to invent. The advanced learning goal of the instruction was to learn about the concept of the buoyant force and how the magnitude of the buoyant force is determined. This advanced learning goal was a test of whether invention activities could benefit the learning of more distal concepts around the same topic.

\section{Tests}

All participants completed a pre-test at the beginning of the study, and a post-test after reading the instruction. The two treatment conditions (CC and SIM) had an extra midtest after finishing the invention activity and before reading the instructional text.

The pre-test consisted of three questions: two questions surveying participants' prior experience with physics and familiarity with buoyancy on a 5-point scale; and an openended question to write a general rule to predict floating and sinking of objects in water.

The mid-test contained five questions on whether a particular object would float in water, an open-ended question on what the most difficult concept for teaching buoyancy to someone else would be, and a final question asking participants to write a general rule for determining whether objects in fluids would float or sink.

The post-test included 27 multiple-choice questions and seven open-ended questions. The multiple-choice questions were divided in two groups: 1 . density questions $(n=13)$ requiring a comparison between an object's density and fluid density to determine whether the object would sink or float; 2. buoyancy questions $(n=14)$ requiring participants to determine the magnitude of the buoyant force in different situations. Similar to pre- and mid-tests, one of the openended questions in the post-test asked participants to write the general rule for floating and sinking of objects in the fluids.

\section{RESULTS}

\section{A. Scoring}

Each of the pre-test multiple-choice questions were scored on a scale of 1 ("have taken no relevant course" or "not at all familiar") to 5 ("have taken a relevant college course" or "very familiar"). The multiple choice questions on the mid and post-test were graded 0 for incorrect and 1 for correct, as were the rules written in the pre-, mid-, and post-tests. A correct density rule would incorporate a 
correct comparison between object density and fluid density for predicting floating and sinking.

\section{B. Pre-test}

There was no significant difference across conditions in terms of: prior experience about and familiarity with the topic of buoyancy, $F(2,76)=0.33, p=0.72$; or the number of participants who wrote a correct rule in their pre-test, $\chi^{2}(2,79)=0.31, p=0.85$.

\section{Post-test}

Density score: Figure 2 shows the average post-test scores across density and buoyancy questions for the three conditions. For the density questions, there was a main effect of condition, $F(2,76)=7.01, p=0.002$. Looking at pairwise differences, there was a significant difference between CC and SIM in the average density score $\left(m_{\mathrm{CC}}=11.8, s d_{\mathrm{CC}}=1.41, m_{\mathrm{SIM}}=9.64, s d_{\mathrm{SIM}}=3.50\right)$, $t(6.42)=3.00, p=0.005, d=0.79$. There was, however, no difference between SIM and CTRL conditions $\left(m_{\mathrm{CTRL}}=9.12, s d_{\mathrm{CTRL}}=2.69\right), t(50.3)=0.62, p=0.54$. This result suggests that an invention activity with contrasting cases prior to direct instruction significantly improved participants' learning of the primary learning goal here. However, an invention activity with simulation did not have such an effect on participants' learning.

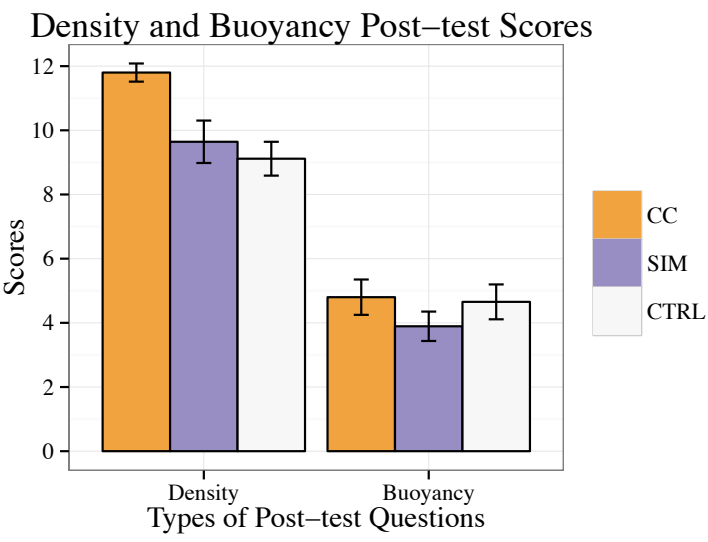

FIG 2. Density and buoyancy post-test scores for the three conditions. Error bars on all graphs are 1 standard error.

Buoyancy score: For buoyancy questions, there was no difference across the three conditions, $F(2,76)=0.91, p=$ 0.41 (Fig. 2). That is, for this advanced learning goal, there was no benefit of attempting to invent the related density rule.

Post-test rule: There was a difference in percentage of correct post-test rules by condition $(p=0.03)$ : $96 \%$ in the CC condition, $71 \%$ in the SIM condition, and $69 \%$ in the CTRL condition (Fig. 3). Just as with the density post-test questions, SIM and CTRL performed almost similarly and were outperformed by CC.
Given these results, there are two possible ways in which the $\mathrm{CC}$ materials aid learning about density. One possibility is that the $\mathrm{CC}$ invention activity is no better than the SIM invention activity in how much it helps participants understand the density rule, but that $\mathrm{CC}$ better prepares participants to understand the density rule in the instructional text. However, the data from mid-test supports a second possibility: the contrasting cases helped participants better understand the density rule even before reading the instructional text.

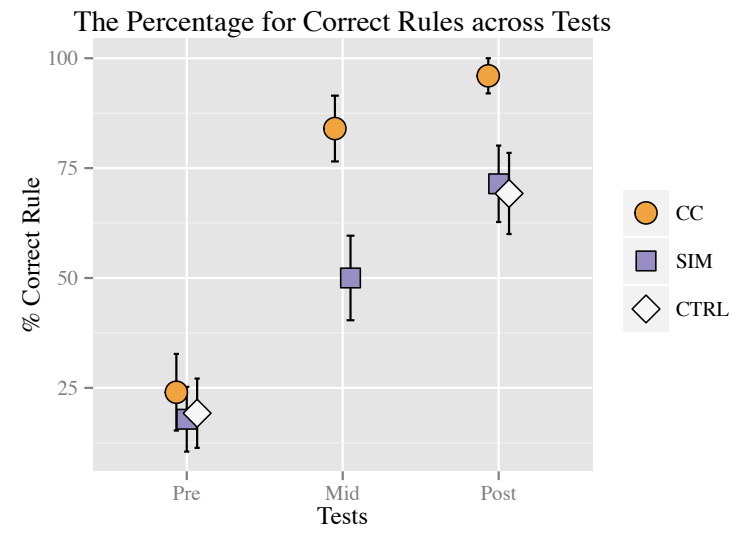

FIG 3. Percentage of participants with correct rules across pre-, mid-, and post-tests for the three conditions.

\section{Mid-test}

Mid-test score: For the five mid-test questions, as Fig. 4 shows, participants in the CC condition scored significantly higher, $U=446, p=0.03$ (out of maximum score of five: $\left.m_{\mathrm{CC}}=4.72, s d_{\mathrm{CC}}=0.93, m_{\mathrm{SIM}}=4.04, s d_{\mathrm{SIM}}=1.57\right)$.

Mid-test Rule: Although CC performed better on the mid-test about the floatation of different objects in water, we were also interested in the effect of CC and SIM on discovering the full density rule, which addresses variation in fluid density as well as object density. Therefore, we also looked at the mid-test rules participants wrote in the treatment conditions; $84 \%$ of participants in the $\mathrm{CC}$ condition wrote a correct rule at mid-test, whereas in the SIM condition only $50 \%$ did $(p=0.02)$, as shown in Fig. 3 . Both mid-test scores and mid-test rules indicate that even before the instructional text, there was a difference in how many participants could state the correct density rule.

Mid-test rule components: To invent a correct rule it is necessary, though not sufficient, for participants to notice both object density and fluid density. Between $\mathrm{CC}$ and SIM, there was no significant difference in the number of participants who included object density in their rule $(68 \%$ in SIM compared to $76 \%$ in CC). However, significantly fewer participants in the SIM condition mentioned fluid density in their invented rule (54\% in SIM compared to $92 \%$ in CC), $\chi^{2}(1,53)=7.81, p=0.005$. This difference could be the result of the $\mathrm{CC}$ condition being provided with 
the contrasting cases that varied in both fluid density and object density, while in the SIM condition participants generated the cases themselves and may not have attended to fluid density.

Using recordings of participants' exploration with the simulation, we divided participants in the SIM condition into participants who varied the effect of fluid density using the "playground" tab of the simulation $(n=13)$ and those who used the "playground" tab, but did not vary the fluid density $(n=9)$. Participants who "checked fluid density" had significantly higher density scores in the post-test $\left(m_{\text {checked }}=11.0, s d_{\text {checked }}=3.49, \quad m_{\text {not checked }}=8.44\right.$, $\left.s d_{\text {not checked }}=3.05\right), U=27, p=0.03$. The same pattern was observed in the invented rules (Fig. 4). For participants in SIM who checked fluid density, there was no significant difference in the percentage of correct rules compared to $\mathrm{CC}$ in both mid-test $(p=0.23)$, and post-test $(p=0.27)$. However, for participants who did not check the fluid density, in both tests, this percentage was significantly lower than $\mathrm{CC}(p=0.002, p=0.002)$. This suggests that SIM is not inherently worse than $C C$, but rather that its value depends on successful navigation of the complexity of the simulation to identify all parameters relevant to the phenomenon. CC may help students manage the complexity with a small set of cases to point out all the key features, such as fluid density.

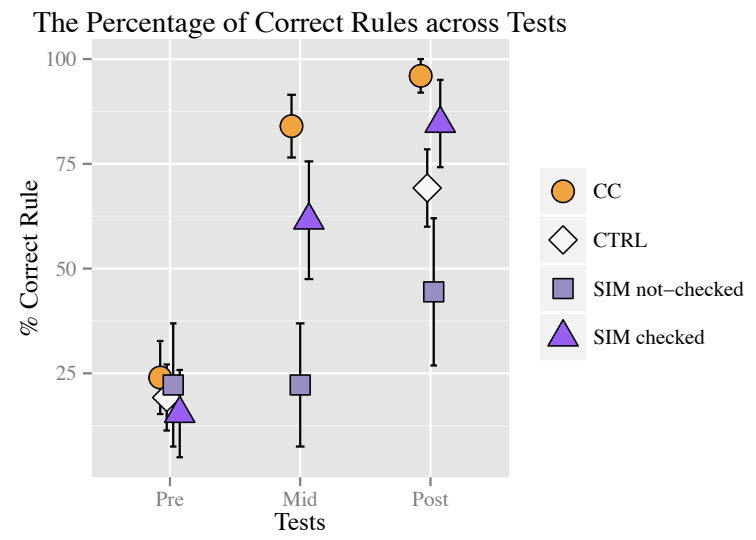

FIG 4. Percentage of participants with correct rules across pre-, mid-, and post-tests for all the conditions, dividing the SIM condition into participants who checked fluid density and ones who did not.

\section{CONCLUSIONS}

This study shows that, in a short instructional sequence, a contrasting cases invention activity leads to better learning of a physics rule than inventing with an interactive simulation. Though it allows for more autonomy in exploring the phenomenon, free exploration of the many parameters of the simulation increases the possibility that students may fail to incorporate a crucial feature into their rules. Missing essential features during the invention activity can affect further learning from direct instruction.

Nevertheless, the results of this study should not be interpreted as an argument against interactive simulations, but rather as an argument for careful attention to the level of guidance we provide. Adams et al. argued for a "sweet spot" of productive guidance for simulation use, where excessive guidance can lead students to follow directions rather than engage in deep exploration, while lack of any guidance may lead to failure for complex simulations [6]. In our study, the learning benefit of exploring certain key features, such as fluid density, reiterates the need for some guidance that helps students attend to such features in complex environments. Had we guided participants' attention towards the fluid density, as we did by varying fluid density in the contrasting cases, we might have maximized the benefit of inventing with a simulation.

A future research direction could investigate the level of scaffolding that best supports exploration and learning with simulations. Crucially, interactive simulations are also hypothesized to have many learning benefits that were not measured in this study. The interactive nature of these environments with immediate feedback gives participants the means for scientific experimentation and hypothesis testing. Future work will explore how we can capitalize on the unique affordances of simulations for scientific inquiry while making sure that students are prepared for future learning of the canonical principles of physics.

\section{ACKNOWLEDGEMENTS}

The authors would like to acknowledge Wieman's and Schwartz's research groups for their valuable feedback. This work is supported by the Gordon and Betty Moore Foundation.
[1] D. L. Schwartz, and T. Martin, Cogn. Instr. 22, 129 (2004).

[2] D. L. Schwartz, C. C. Chase,, M. A. Oppezzo, and D. B. Chin, J. Educ. Psych. 103, 759 (2011).

[3] C. E. Wieman, W. K. Adams, and K. K. Perkins, Science 322, 682 (2008).

[4] N. R. Hallinen, K. P. Blair, D. B. Chin, and D. L. Schwartz, in Proceedings of the International Conference of the Learning Sciences, Boulder, 2014, edited by J.L. Polman, E.A. Kyza, D.K. O'Neill, I. Tabak, W.R. Penuel, S.A. Jurow, K. O'Connor, T. Lee, and L. D'Amico. (University of Colorado, Boulder, 2014), p. 31.

[5] https://phet.colorado.edu/en/simulation/buoyancy, retrieved 6/29/2015.

[6] W. K. Adams, A. Paulson, and C. E. Wieman, AIP Conf. Proc. 1064, 59 (2008). 